\title{
Horosphere slab separation theorems in manifolds without conjugate points
}

\author{
Sameh Shenawy
}

Correspondence: drshenawy@mail.com

Department of Mathematics, Modern Academy, Maadi, Cairo, Egypt

\begin{abstract}
Let $\mathcal{W}^{n}$ be the set of smooth complete simply connected $n$-dimensional manifolds without conjugate points. The Euclidean space and the hyperbolic space are examples of these manifolds. Let $W \in \mathcal{W}^{n}$ and let $A$ and $B$ be two convex subsets of $W$. This note aims to investigate separation and slab horosphere separation of $A$ and $B$. For example, sufficient conditions on $A$ and $B$ to be separated by a slab of horospheres are obtained. Existence and uniqueness of foot points and farthest points of a convex set $A$ in $W \in \mathcal{W}$ are considered.
\end{abstract}

Keywords: Horosphere separation, Slab horosphere separation, Manifolds without conjugate points, Separation of convex sets

AMS Subject Classification: Primary; 52A10, 52A20; Secondary 52A55

\section{An introduction}

Let $\omega$ be a unit tangent vector to a smooth complete simply connected manifold without conjugate points $W \in \mathcal{W}^{n}$ at a point $p \in W$. Let $\alpha$ be the unique geodesic with tangent $\omega=\alpha^{\prime}(0)$ and $p=\alpha(0)$. The Busemann function $b_{\omega}: W \in \mathcal{W}^{n} \rightarrow \mathbb{R}$ is defined by

$$
b_{\omega}(x)=\lim _{t \rightarrow \infty}[t-d(x, \alpha(t))],
$$

where $d$ is the distance function. The right hand side is well-defined and the Busemann function $b_{\omega}$ is smooth in a complete simply connected manifold without conjugate point $W \in \mathcal{W}^{n}$ whereas $b_{\omega}$ is at least $C^{2}$ given that $W$ has no focal points(see [1, Theorem 2]). The level set of a Busemann function, that is $b_{\omega}^{-1}(0)$, is called a horosphere $H_{\omega}(p)$ where $p=\alpha(0)$. Likewise, the open and the closed horoballs in $W \in \mathcal{W}^{n}$ are defined as the sets $D_{\omega}(p)=b_{\omega}^{-1}((0, \infty))$ and $\bar{D}_{\omega}(p)=b_{\omega}^{-1}([0, \infty))$ respectively. Let $\alpha(t)$ be the geodesic passing through a point $p \in W \in \mathcal{W}^{n}$ with $\alpha^{\prime}(0)=\omega$. It is well-known that the horosphere $H_{\omega}(p)$ is the limit of the geodesic spheres $S(\alpha(t), t)$ passing through $p=$ $\alpha(0)$ and having center $\alpha(t)$ as $t \rightarrow \infty$. The horospheres $H_{u}(p), u=\alpha^{\prime}(0), p=\alpha(0)$ and $H_{v}(q) v=\alpha^{\prime}(a), q=\alpha(a)$ are called co-directional or parallel horospheres and parallel horospheres touch each other at infinity. Notice that the horopsheres $H_{\omega}(p)$ and $H_{-\omega}(p)$ have $p$ as their unique common point; otherwise, they coincide. Hyperplanes are horospheres in the Euclidean space $E^{n}$. The horosphere $H_{\omega}(p)$ with a given direction $\omega$ and a given point $p$ is unique. Finally, the horospheres, as a level surfaces of a Busemann function, are equidistant family of surfaces whose orthogonal trajectories are geodesics.

(c) The Author(s). 2019 Open Access This article is distributed under the terms of the Creative Commons Attribution 4.0 International License (http://creativecommons.org/licenses/by/4.0/), which permits unrestricted use, distribution, and reproduction in any medium, provided you give appropriate credit to the original author(s) and the source, provide a link to the Creative Commons license, and indicate if changes were made. 
It is noted that, thanks to the well-known Hopf-Rinow theorem, there is a length minimizing geodesic segment joining each pair of points in a complete connected Riemannian manifold $W$. If, in addition, $W$ is simply connected and has no conjugate points, then the exponential map is a covering map and each pair of points is joined by a unique and hence minimal geodesic(see Section 10.7 of [2]). Finally, a set $A$ of $W \in \mathcal{W}^{n}$ is compact if and only if it is closed and bounded. All manifolds with negative curvature are members of $\mathcal{W}^{n}$. For example, the hyperbolic Poincare upper half-plane model

$$
H^{2}=\left\{(x, y) \in \mathbb{R}^{2}: y>0\right\},
$$

equipped with the metric $g_{11}=g_{22}=y^{-2}$ and $g_{12}=0$ lies in $\mathcal{W}^{2}$ (see [3] for more details); however, the unit sphere $S^{2}$ does not lie in $\mathcal{W}^{2}$ since all antipodal points are conjugate points.

A subset $A$ of $W \in \mathcal{W}^{n}$ is convex if the geodesic segment $[p q]$ joining any two points $p, q \in A$ lies in $A$. Three different definitions of convex sets in general Riemannian manifolds were studied in [4]. The whole manifold $W$ geodesics are all convex sets. Also, open and closed geodesic balls of manifolds with negative curvature are convex sets. On the other hand, the union of two different geodesics is not convex and the complement of a convex set is not necessarily convex. Note that the existence and uniqueness of geodesic segments in these manifolds is trivial; however, for example, the whole sphere $S^{n}$ is not convex since antipodal points have many minimal geodesic segments joining them. Convex functions are also deeply studied in Riemannian geometry (the reader is referred to [5] for a detailed study of convex functions on manifolds with negative curvature).

Let $p$ be a point in a complete simply connected manifold without conjugate point $W \in$ $\mathcal{W}^{n}$. The point $p$ has a foot point $f$ in subset $A$ of $W$ if the distance function $l: A \rightarrow \mathbb{R}$ defined by $l(x)=d(p, x), x \in A$ attains its minimum at $f$. The point $p$ is said to have a farthest point $F$ in $A$ if the function $l$ attains its maximum at $F[6,7]$. The geodesic ray starting at $p$ and passing through $q$ is denoted by $R(p q)$, and the entire geodesic passing through them is denoted by $G(p q)$.

Convex sets, foot, and farthest points play a very important role in both convex analysis and optimization (see for example [8-10] and references therein). Generalizations and extensions of convex sets and their separation and supporting surfaces are of particular interest $[11,12]$. Each pair of points in a simply connected smooth Riemannian manifold without conjugate points has a unique and hence minimal geodesic joining them whereas manifolds without focal points has convex geodesic spheres [13-17]. It is well-known that the class of complete simply connected manifolds without focal points is a proper subclass of $\mathcal{W}^{n}$. Manifolds with non-positive sectional curvatures have no focal points [18-22]. Horospheres and totally geodesic hypersurfaces in $W \in \mathcal{W}^{n}$ play a significant role in defining both supporting and separation theorems for convex sets.

In this note, the concepts of separation and horosphere slab separation of convex sets are studied in $W \in \mathcal{W}^{n}$. Sufficient conditions for two disjoint closed convex sets to be separated by a slab of horosheres are given. Foot and farthest points of a convex set in $W \in \mathcal{W}^{n}$ are considered.

\section{Foot and farthest points of a convex set $A$ in $\boldsymbol{W} \in \mathcal{W}^{n}$}

This section is devoted to the study of foot and farthest points of a convex set $A$ in $W \in$ $\mathcal{W}^{n}$. The geodesic sphere with the center at $p$ and radius $r$ is denoted by $S(p, r)$ and the 
corresponding open and closed geodesic balls are denoted by $B(p, r)$ and $\bar{B}(p, r)$.

Let us begin with the following simple but important result.

Proposition 1 In a complete simply connected Riemannian manifold without conjugate points $W \in \mathcal{W}^{n}$, the following statements are true.

1. If $\alpha$ is the unique geodesic parameterized by arc length with $\alpha(0)=x, \alpha^{\prime}(0)=\omega$, and $\alpha(r)=p$, then the geodesic segment $[x y]$ intersects $B(p, r)$ for any $y \in D_{\omega}(x)$.

2. Let $B(p, r)$ be a geodesic ball with the center at $p$ and radius $r$, then any point $x \neq p$ has a foot point $f=S(p, r) \cap R(p x)$ in $S(p, r)$.

Theorem 1 Let $W \in \mathcal{W}^{n}$ be a manifold without focal points and $A$ be a non-empty closed convex subset of $W$. Then, each point $p$ of $W$ has a unique foot point.

Proof Since $W$ has no focal points, the geodesic ball $\bar{B}(p, r)$ is convex and hence $A \cap$ $\bar{B}(p, r)$ is either convex or empty. The result follows easily if $p \in A$. So, assume that $p \notin A$. Let $q$ be in $A$ and $r=d(p, q)$. It is clear that $G=A \cap \bar{B}(p, r)$ is a closed no-empty convex subset of $\bar{B}(p, r)$. Then, $G$ is compact. Define the real-valued function $f(x)=d(p, x)$ on G. $f$ is continuous function and consequently attains its minimum at a point $f$ in $A$. To show that $f$ is unique, assume that $p$ has two foot points $f_{1}$ and $f_{2}$ in $A$. Then, the closed ball $\bar{B}(p, r)$ touches $A$ twice where $d\left(p, f_{1}\right)=d\left(p, f_{2}\right)=r$ (see Fig. 1$)$. The open segment $\left(f_{1} f_{2}\right)$ is contained in $G$ since $G$ is convex, and so both $f_{1}$ and $f_{2}$ are not foot points of $p$. This contradiction shows that $f$ is unique(see Fig. 1).

Corollary 1 Let $W \in \mathcal{W}^{n}$ be a manifold without focal points and $A$ be a non-empty closed convex subset of $W$ and let $p \notin A$. Iff is a foot point of the point $p$, then $f$ is the unique foot point of any $q \in(p f)$ in A. Likewise, if $p$ has a farthest point $F$ in $A$ from and $p \in(F q)$ for some point $q$, then $q$ has $F$ as its unique farthest point in $A$.

The following theorems represent two analogous results to the above ones.

Theorem 2 Let $W \in \mathcal{W}^{2}$ and $A$ be a non-empty convex subset of $W$. If $p \in W \backslash A$ has a foot point $f$ in $A$, then $f$ is a foot point in $A$ for every point of $R(f p)$.

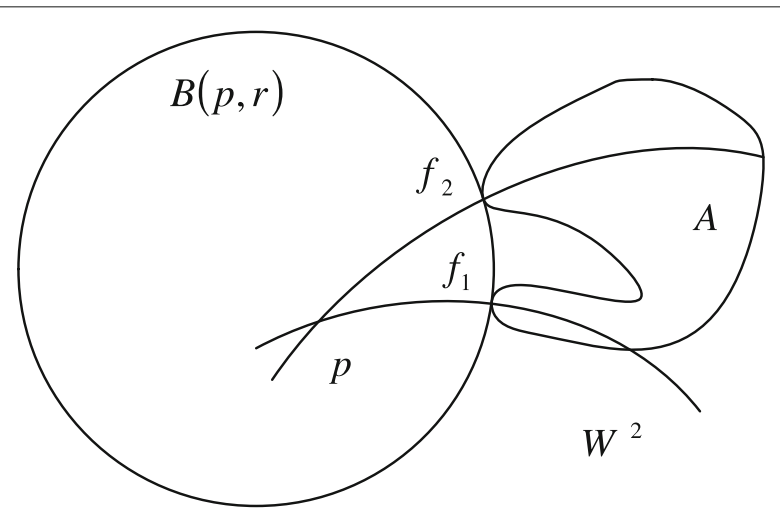

Fig. 1 Uniqueness of foot points in a convex set 
Proof There is a geodesic $\gamma$ supporting $A$ at $f$ since $A$ is convex. Let $\alpha$ be the unique geodesic with $f=\alpha(0)$ and $p=\alpha(r)$. Let $H_{v}(f)$ be the horosphere with $v=\alpha^{\prime}(0)$. The closed ball $\bar{B}(q, l), l=d(q, f)$ is contained in $\bar{D}_{v}$ for any point $q \in R(f p)$. Thus, $f$ is the unique foot point of $q$ in $A$ (see Fig. 2).

Theorem 3 Let $W \in \mathcal{W}^{n}$ and $A$ be a non-empty compact subset of $W$. Then, every point $p \notin A$ has a farthest point in $A$.

Proof The function $l: A \rightarrow \mathbb{R}$ defined on $A$ by $l(x)=d(p, x)$ for every $x \in A$ is a realvalued continuous function. Since $A$ is compact, $l$ attains its maximum at point in $A$ say $F$. Thus, $F$ is the farthest from $p$ in $A$.

\section{Separation of convex sets in $\boldsymbol{W} \in \mathcal{W}^{n}$}

Separation of two convex sets in the Euclidean space $E^{n}$ is widely used in optimization. The most well-known separation theorem says that any two non-empty disjoint convex sets in the Euclidean space are separated by a hyperplane. There are more restrictive separation theorems for different types of convex and non-convex sets.

In Riemannian geometry, it is natural to ask the following question. What is the best candidate for a hyperplane in separation theorems? Horospheres in complete simply Riemannian manifolds without conjugate points play this significant role in separation of two convex sets.

A slab in the Euclidean space is the region bounded by two parallel hyperplanes. Here, a slab of horospheres along a geodesic $\alpha$ is the region bounded by $H_{\alpha^{\prime}(0)}$ and $H_{\alpha^{\prime}(r)}$. It is denoted by $S_{\alpha}[0, r]$ (see Fig. 3). Two sets $A$ and $B$ are said to be separated by a slab of horospheres if there is a geodesic $\alpha$ such that $A$ and $B$ lie in two different sides $S_{\alpha}[0, r]$.

Theorem 4 Let $W \in \mathcal{W}^{n}$ be a complete simply connected Riemannian manifold without conjugate points and $A, B$ be two non-empty disjoint convex subsets of $W . A$ and $B$ are

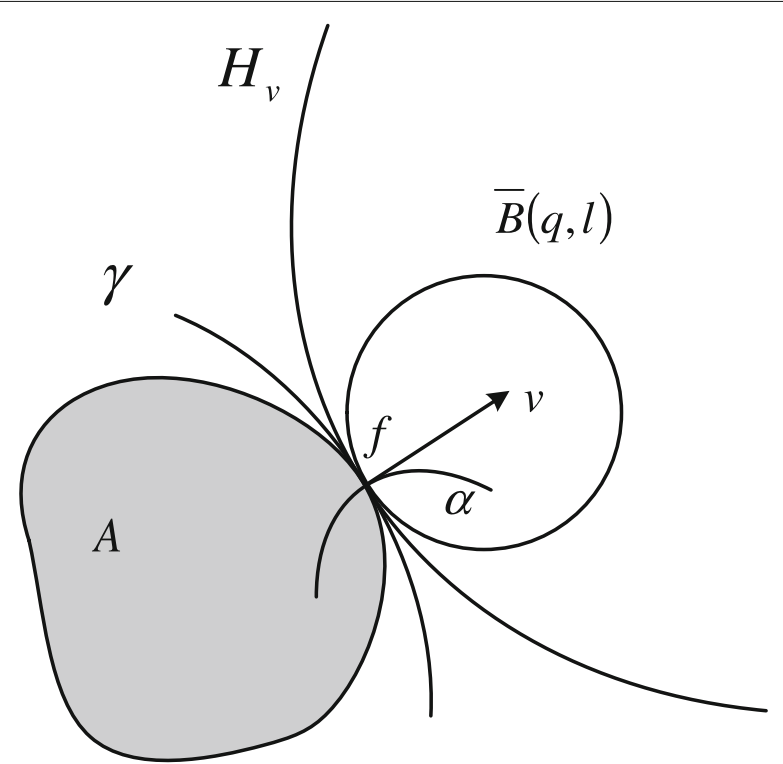

Fig. 2 Supporting at foot points 


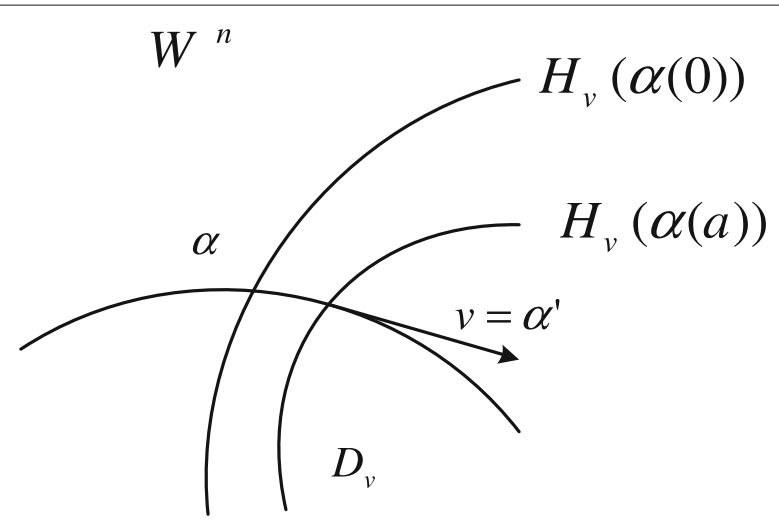

Fig. 3 A slab

separated by a slab of horospheres if one of them is bounded and the second is supported by a horosphere at every boundary point.

Proof Assume that $A$ is bounded and $B$ has a supporting horosphere at each point $p \in$ $\partial A$. The real-valued function $l$ defined on $A$ by $l(x)=d(x, B)$ for every $x \in A$. $l$ attains its minimum value at a point $p \in A$ since $A$ is compact and $l$ is continuous. The point $p$ has a foot point $f \in B$ where $p \neq f$. B has a supporting horosphere $H_{\alpha^{\prime}(r)}(q)$ where $\alpha$ be the unique geodesic with $\alpha(0)=q$ and $\alpha(r)=p$. ince $B$ is convex and hence supported by a totally geodesic hypersurface at $q$ that separates $B$ and $H_{\alpha^{\prime}(0)}$. Now, the slab $S_{\alpha}[0, r]$ separates $A$ and $B$ (see Fig. 4).

Corollary 2 Let $W \in \mathcal{W}^{n}$ and $A, B$ be two non-empty disjoint convex subsets of $W . A$ and $B$ are strictly separated if one of them is compact and the other one is closed.

The Euclidean version of the above theorem is as follows (see Theorem 7.6 in [12]). Note that the term convex is replaced by the term compact.

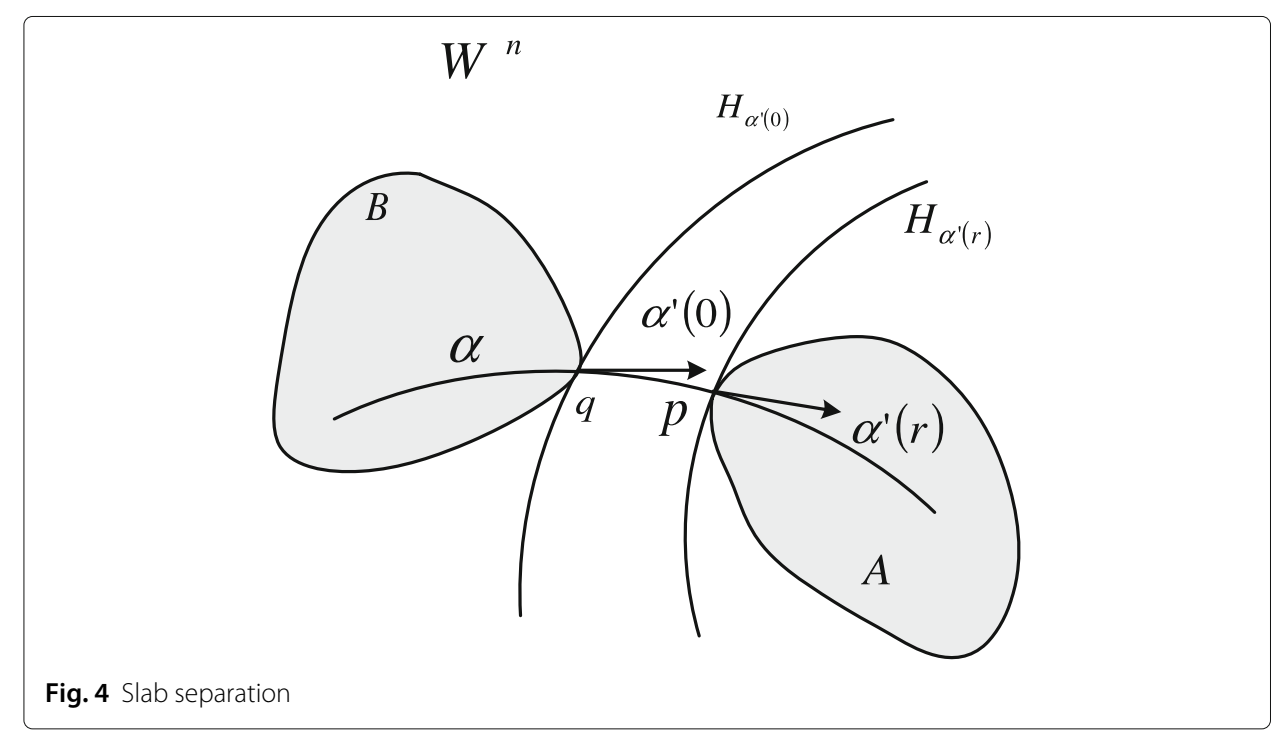


Theorem 5 Let A, B be two non-empty disjoint compact subsets of the Euclidean space $E^{n} . A$ and $B$ are strictly separated by a slab if and only if for each set $T$ of $n+2$ or fewer points of $A \cup B$ the sets $A \cap T$ and $B \cap T$ are separated by a slab.

\section{Acknowledgments}

The author would like to thank the referee(s) for the insightful comments that improve the paper quality.

\section{Authors' contributions}

The author read and approved the final manuscript.

Funding

Not applicable.

Availability of data and materials

Not applicable.

\section{Competing interests}

The author declares that he has no competing interests.

Received: 27 March 2019 Accepted: 26 August 2019

Published online: 18 September 2019

\section{References}

1. Eschenburg, J. H.: Horospheres and the stable part of the geodesic flow. Math. Z. 153(3), 237-51 (1977)

2. Hicks, NJ.: Notes on differential geometry. Van Nostrand, New York (1965)

3. Carmo, MP.: Riemannian geometry. Birkhäuser, Berlin (1992)

4. Alexander, S.: Local and global convexity in complete Riemannian manifolds. Pac. J. Math. 76(2), 283-9 (1978)

5. Bishop, R. L., O'Neill B.: Manifolds of negative curvature. Trans. Am. Math. Soc. 145, 1-49 (1969)

6. Beltagy, M., Shenawy, S.: Sets with zero-dimensional kernels. Int. J. of Mod. Math. 4(2), 163-8 (2009)

7. Shenawy, S.: Tietze-type theorem in 2-dimensional Riemannian manifolds without conjugate points. Bak. J. Geom. Appl. 16(2), 133-137 (2011)

8. Bacàk, M.: Convex analysis and optimization in Hadamard spaces. Vol. 22. Walter de Gruyter GmbH \& Co KG, Berlin (2014)

9. Boyd, S., Vandenberghe, L.: Convex optimization. Cambridge university press, Cambridge (2004)

10. Nesterov, Y.: Lectures on convex optimization. Vol. 137. Springer, New York (2018)

11. Deumlich, R., Elster, K.-H., Nehse, R.: Recent results on separation of convex sets. Optimization. 9(2), $273-296$ (1978)

12. Lay, S.: Convex sets and their applications. Wiley, New York (1982)

13. Aquino, C. P., Baltazar, H. I., de Lima, H. F.: Characterizing horospheres of the hyperbolic space via higher order mean curvatures. Differ. Geom. Appl. 62, 109-119 (2019)

14. Goto, M. S.: Manifolds without conjugate points. J. Differ. Geom. 13, 341-359 (1978)

15. Liu, F, Wang, F: Entropy-expansiveness of geodesic flows on closed manifolds without conjugate points. Acta Math. Sin. Engl. Ser. 32, 507-520 (2016)

16. Liu, F., Zhu, X.: The transitivity of geodesic flows on rank 1 manifolds without focal points. Differ. Geom. Appl. 60, 49-53 (2018)

17. Ranjan, A., Shah, H.: Convexity of spheres in a manifold without conjugate points. Proc. Indian Acad. Sci.-Math. Sci. 112(4), 595-599 (2002)

18. Kobayashi, S: Riemannian manifolds without conjugate points. Annali di Matematica. 53(1), 149-155 (1961)

19. Benjamini, I., Eldan, R.: Convex hulls in the hyperbolic space. Geom. Dedicata. 160, 365-371 (2012)

20. Morse, M, Hedlund, G. A.: Manifolds without conjugate points. Trans. Am. Math. Soc. 51, 362-386 (1942)

21. O'Sullivan, J. J.: Manifolds without conjugate points. Math. Ann. 210(4), 295-311 (1974)

22. Ruggiero, R. O.: A note on the divergence of geodesic rays in manifolds without conjugate points. Geom. Dedicata. 134, 131-138 (2008)

\section{Publisher's Note}

Springer Nature remains neutral with regard to jurisdictional claims in published maps and institutional affiliations. 